Théologiques

Théologiques

\title{
Mémoire et prégnance des lieux dans la structuration de l'islam en Martinique
}

\section{Liliane Kuczynski}

Volume 15, numéro 1, 2007

Mémoires et usages religieux de l'espace

URI : https://id.erudit.org/iderudit/017637ar

DOI : https://doi.org/10.7202/017637ar

Aller au sommaire du numéro

\section{Éditeur(s)}

Faculté de théologie et de sciences des religions, Université de Montréal

\section{ISSN}

1188-7109 (imprimé)

1492-1413 (numérique)

Découvrir la revue

\section{Citer cet article}

Kuczynski, L. (2007). Mémoire et prégnance des lieux dans la structuration de l'islam en Martinique. Théologiques, 15(1), 69-93.

https://doi.org/10.7202/017637ar
Résumé de l'article

En Martinique, l'islam est apparu dans les années 1980. Représenté d'abord par des migrants venus d'Afrique de l'Ouest et du Maghreb, il a gagné progressivement des Martiniquais qui se sont convertis tandis que d'autres, notamment des commerçants palestiniens présents dans les îles depuis longtemps, reprenaient une pratique religieuse qu'ils avaient abandonnée. La question de l'origine et la mémoire des lieux jouent un rôle essentiel dans les essais de structuration de l'islam dans les îles et dans la séparation en différents groupes. On identifiera trois références antagonistes, variables dans leur efficacité et leurs effets, qui orientent de façon différente selon les groupes tant l'agencement des lieux que les rituels et les comportements des fidèles : la référence à l'arabité et à un terroir « originel » oriental; la référence (ambiguë) à l'Afrique; la référence au terroir antillais.
Tous droits réservés @ Faculté de théologie et de sciences des religions, Université de Montréal, 2007
Ce document est protégé par la loi sur le droit d'auteur. L'utilisation des services d'Érudit (y compris la reproduction) est assujettie à sa politique d'utilisation que vous pouvez consulter en ligne.

https://apropos.erudit.org/fr/usagers/politique-dutilisation/ 


\title{
Mémoire et prégnance des lieux dans la structuration de l'islam en Martinique
}

\author{
Liliane KUCZYNSKI \\ Laboratoire d'anthropologie urbaine \\ Centre national de la recherche scientifique, France
}

Évoquer les musulmans en Martinique signifie qu'on se situe d'emblée dans le cadre d'une île marquée par de multiples mobilités et migrations récentes. Rappelons pour mémoire la mobilité des Martiniquais eux-mêmes, vers l'Amérique du Nord et la France, destination quasi obligée à une certaine époque (celle de l'existence du BUMIDOM ${ }^{1}$ ). Un mouvement de retour s'est amorcé dans les années 1980; on verra le lien entre le retour des Martiniquais dans leur île et le développement de l'islam en Martinique. Mais d'autres courants migratoires ont joué un rôle plus nettement déterminant dans l'implantation de cette pratique religieuse. On présentera d'abord la population musulmane de la Martinique et son histoire; puis, on tentera de définir et d'analyser les mémoires sous-jacentes ou conscientes qui orientent lieux, hommes et rituels ${ }^{2}$.

\section{Présentation de la population musulmane de la Martinique}

Le nombre des musulmans vivant en Martinique peut être évalué à environ 500 personnes, nombre qui représente moins de $1 \%$ de la population totale de l'île ${ }^{3}$. Mais ce chiffre doit être considéré comme très approximatif pour plusieurs raisons: l'extrême mobilité physique de cette population, toutes origines confondues; la mobilité religieuse, assez fréquente dans la Caraïbe (certains convertis adhèrent à l'islam de façon provisoire ou conjoncturelle); le lien extrêmement ténu ou très sporadique que certains musulmans

1. Bureau pour le développement des migrations dans les départements d'Outre-Mer, organisme public qui, de 1963 à 1981, fut chargé d'accompagner l'émigration des habitants des territoires d'Outre-Mer vers la métropole.

2. Les analyses qui suivent résultent d'enquêtes de terrain menées en Martinique de 1999 à 2004.

3. 391500 habitants en 2005 (Planson 2005).

(C) Revue Théologiques 2007. Tout droit réservé. 
d'origine entretiennent avec les lieux de culte; l'absence (voire le refus) de revendication d'une identité musulmane de la part de certains autres. Le chiffre de 500 est donc extensif et comptabilise des personnes représentant de multiples niveaux et formes d'adhésion à l'islam. Il résulte d'une estimation faite en 1999, lors de la première fête musulmane célébrée dans l'espace public — événement qui a attiré plus de sympathisants que n'en comptent habituellement les divers cercles de musulmans de l'île.

La composition de cette population musulmane est pour une très grande part d'origine étrangère. On peut à travers elle retracer une partie des courants migratoires et de la diversité sociale et culturelle de l'île. Car si selon les statistiques le nombre d'étrangers vivant en Martinique est très faible, sa population n'en est pas moins très brassée et d'origines multiples; l'île est encore aujourd'hui un lieu où l'on vient tenter sa chance parfois de manière très provisoire.

Les musulmans les plus anciennement présents en Martinique sont des Palestiniens presque tous originaires de Ramallah et sa région. Arrivés sur l'île dans les années 1930-1940, ils s'y sont arrêtés un peu par hasard sur la route de l'Amérique du Sud. Ils font partie d'un courant migratoire débuté à la fin du XIX siècle, lorsque, pour des raisons économiques et politiques (notamment le renforcement du contrôle étatique turc), des ProcheOrientaux arabophones ont quitté en grand nombre les territoires sous administration ottomane ${ }^{4}$. Certaines familles sont donc présentes dans l'île depuis trois, voire quatre générations; les plus jeunes y sont nées, y ont acquis la nationalité française et sont aussi bien créolophones qu'arabophones. Cette immigration venant de Palestine ne s'est jamais réellement interrompue et a été plus ou moins faiblement entretenue par d'autres événements économiques et politiques plus récents affectant le Proche-Orient: guerre des Six Jours, intifada. D'abord colporteurs, puis sédentarisés dans le centre de Fortde-France, les Palestiniens se sont spécialisés dans le commerce des vêtements ${ }^{5}$. Un petit nombre d'entre eux sont devenus des entrepreneurs prospères. De tous les musulmans de Martinique, ce sont eux qui détiennent le pouvoir économique. Ils sont nommés depuis longtemps «Syriens » par les Martiniquais - vaste catégorie englobant tous les commerçants levantins, quelle que soit leur origine ou leur religion ${ }^{6}$; ce terme désigne

4. Voir l'analyse des causes de cette émigration levantine vers l'Amérique du Sud dans Cuche 2001. L'auteur note que pour les migrants, l'Amérique était perçue «comme une destination indifférenciée » (91).

5. Mais le colportage est toujours pratiqué par certains.

6. De même, ils sont désignés en Amérique latine du nom global de «turcos », en raison de leur provenance de l'Empire ottoman (Cuche 2001, 99). 
aussi actuellement les marchands maghrébins. Les autres musulmans les particularisent sous le terme d' "Arabes ", aussi utilisé pour les Maghrébins. Ce qui caractérise les Palestiniens est le maintien fort de liens avec leur région d'origine et de nombreux va-et-vient entre Martinique et Proche-Orient.

Le deuxième groupe important mais historiquement plus récent de musulmans vivant en Martinique est constitué d'Africains de l'Ouest, originaires principalement de Guinée et du Sénégal, et de quelques Maliens. Arrivés dès la fin des années 1970, ils sont pour la plupart marabouts ou marchands ambulants; un très petit nombre d'entre eux exercent des professions libérales. La plupart ont fréquenté l'école coranique dans leur jeune âge. Toutes les confréries religieuses existant en Afrique de l'Ouest sont représentées en Martinique ${ }^{7}$, mais certains ne s'en revendiquent pas, notamment les cadres. Comme pour les Palestiniens, la circulation est intense entre lieux d'origine et Antilles, mais aussi d'autres destinations: métropole, Guyane, etc. Un petit nombre d'Africains est sédentarisé en Martinique en raison de mariages contractés avec des Martiniquaises.

D'autres musulmans vivent également en Martinique pour un temps plus ou moins long, certains finissant par se sédentariser, d'autres séjournant dans l'île le temps d'une mission, d'un chantier, d'un poste, d'une tentative de trouver sa place: des Maghrébins, quelques Turcs, Comoriens et Réunionnais, ainsi que des métropolitains convertis à l'islam. Certains sont mariés ou vivent avec des femmes martiniquaises. On trouve parmi eux des professions libérales, des techniciens, des commerçants itinérants, des restaurateurs, des artisans qui fabriquent des objets proposés aux touristes, des chômeurs qui survivent en vendant des babioles ou des lots de vêtements.

On peut remarquer que, contrairement à la Guadeloupe, la population musulmane de la Martinique ne comprend aucun originaire du souscontinent indien.

Mais outre ces musulmans d'origine étrangère et ayant, à des degrés divers, un lien culturel ancien avec l'islam, une part croissance est constituée de Martiniquais convertis à l'islam, soit en Martinique même, soit en France. Les itinéraires les menant à l'islam sont divers et souvent complexes; issus pour la plupart du catholicisme, certains sont ensuite passés par d'autres groupements religieux (adventistes, rastas, témoins de Jéhovah, etc.), dont ils n'abandonnent pas nécessairement toutes les références et valeurs du fait de leur conversion à l'islam ${ }^{8}$. Parmi ces Martiniquais, on

7. Qadriyya, tijaniyya, mouridiyya.

8. Voir à ce sujet des constatations analogues concernant d'autres religions dans les travaux de L. Hurbon (notamment 2000a; 2000b). 
trouve des petits fonctionnaires, des enseignants, des entrepreneurs, des agriculteurs (rastas), des artisans, des techniciens, des chômeurs et RMIstes ${ }^{9}$. La conversion en France a suivi deux chemins principaux: le plus ancien est le contact avec des Africains musulmans connus dans les foyers ${ }^{10}$; plus rarement, la rencontre avec des Maghrébins. Mais la plupart des conversions ont eu lieu sous l'influence du mouvement tabligh, très actif dans les grandes villes françaises et dans les banlieues. Ce mouvement international de propagation de l'islam, né en Inde en 1927, s'est répandu en France dès la fin des années 1960. Il a touché au départ non seulement les jeunes Maghrébins des banlieues, mais aussi les Martiniquais vivant dans ces milieux. Les récits que j'ai pu recueillir montrent que tous ont été séduits par les sessions de plusieurs jours en prières, en exaltation, en vie collective dans les mosquées, ainsi que par les sorties collectives itinérantes pour aller répandre, réveiller la foi. Ils en gardent le souvenir d'une grande intensité et d'une expérience émotionnelle englobante inoubliable.

On voit donc bien se dégager deux caractéristiques majeures de cette population musulmane - expression employée pour éviter les termes «groupe » et "communauté », qui introduisent l'idée trop forte pour mon propos d'une certaine cohésion - : la variété des origines avec une majorité d'étrangers, et l'extrême mobilité. Il en découle un investissement personnel différent dans la constitution d'un espace religieux musulman en Martinique : alors que certains s'enracinent en Martinique, en font le territoire de leur vie religieuse, d'autres, plus nombreux, se sentent de passage quelle que soit la durée de leur séjour sur l'île, et s'impliquent peu dans le développement d'espaces musulmans pérennisés. Les musulmans les plus actifs déplorent souvent vivement l'instabilité, la fragilité des initiatives qui peuvent résulter de cette situation.

J'ai insisté jusqu'à présent sur le caractère exogène de l'islam en Martinique, y compris dans la conversion de certains Martiniquais à l'extérieur de l'île. Mais l'islam a aussi une histoire proprement martiniquaise, qui date de la fin des années 1970.

\section{Développement de l'islam en Martinique}

Les années 1970 ont été marquées par la perte de légitimité et d’hégémonie du catholicisme, conçu comme une religion imposée par les colons et liée

9. C’est-à-dire des bénéficiaires du RMI, revenu minimum d'insertion.

10. Ou, pour un cas, à l'université. 
à l'esclavage, comme une religion inégalitaire impliquant la domination des blancs créoles (Békés). À cette période, on assiste au développement très vif d'Églises issues du protestantisme (évangélisme, pentecôtisme, adventisme, jéhovisme, etc.), comme dans toute l'Amérique latine et désormais en Afrique, et à un accroissement continu des conversions à ces Églises depuis 20 ans. L'Église adventiste du septième jour est le groupe le plus important et le plus prospère (voir Massé 1978; Massé et Poulin 2000). Mais d'autres mouvements religieux que ceux issus du christianisme ont également trouvé place en Martinique, notamment le mahikari, religion prophétique syncrétique importée du Japon. Et dans les années 1980, le rastafarisme se répand dans l'île, qui remet en cause le discours dominant assimilationniste. C'est dans ce bouleversement du système symbolique, dominé jusqu'alors par le catholicisme, et plus profondément dans cette effervescence identitaire qu'on doit situer la prolifération de ces nouveaux groupes, parmi lesquels se trouve aussi l'islam.

C'est en plusieurs étapes que dans ces années 1980 l'islam a trouvé une place en Martinique. Jusqu'à la fin des années 1970, il ne s'agissait que d'une pratique individuelle ou familiale, dans un cadre strictement privé, qui concernait essentiellement des Africains de l'Ouest de passage ou récemment arrivés dans l'île, et très peu les Palestiniens. À cette période n'existaient aucune structure, aucun regroupement, jusqu'à l'intervention d'un marabout charismatique: l'histoire de l'organisation de l'islam en Martinique commence avec lui.

\subsection{Première étape: un marabout charismatique}

Fodé ${ }^{11}$ Marega était un de ces marabouts comme on peut en rencontrer à Paris, recherchant comme bien d'autres une nouvelle clientèle aux Antilles. Homme d'âge mûr, d'origine malienne, il ne représentait certainement que lui-même: un marabout de petite envergure, venu d'abord pour ses propres affaires, avec toujours l'ambivalence, bien connue en Afrique de l'Ouest, entre l'activité de marabout et celle de commerçant. Néanmoins, il était un musulman très convaincu.

C'est d'abord par son activité de guérisseur et de leveur d'infortune, ainsi que par celle de contre-sorcier contre le quimbois, toujours très redouté, qu'il a vite touché une clientèle antillaise. Tout en forgeant son charisme, ces dons qui lui étaient reconnus ont été les prémisses de la

11. Le terme «fodé » désigne en soninké un marabout. 
conversion à l'islam de bien des Antillais, entre les mains desquels il remit, sans insister, des petites brochures, en français, sur les rudiments de l'islam, sur les gestes de la prière.

Cet homme a aussi frappé les Martiniquais par son environnement (chromolithographies de La Mecque et versets calligraphiés en arabe ornant les murs de sa maison), mais surtout par sa prestance et son allure hors du commun. La barbe qu'il portait, la longue robe blanche ${ }^{12}$ dont il était le premier à se vêtir dans l'île leur évoquaient irrésistiblement une imagerie des temps bibliques ${ }^{13}$. Enfin, dernier élément non négligeable dans l'attention particulière portée à ce marabout: son origine africaine. Fodé Marega a sans aucun doute bénéficié de la relation ambivalente des Martiniquais à l'égard de l'Afrique ${ }^{14}$. Tout ce contexte et la personnalité de cet homme fascinant ont contribué à ce qu'un petit nombre de Martiniquais se convertissent à l'islam «dans ses mains».

Le charisme de fodé Marega était très certainement lié à son aura personnelle, et le cadre historique de l'île s'y prêtait: on sait combien les moments de contestation d'une religion dominante, la perte de confiance dans l'institution favorisent l'émergence de personnalités-guides horsnorme. Mais Marega ne s'est pas contenté de ce charisme personnel. Musulman convaincu, il s'est évertué à rassembler les musulmans (déclarés et potentiels) en contactant, un par un ou au travers de leurs associations, les Africains, les Antillais convertis par lui. De même, il a rendu visite à tous les commerçants palestiniens supposés d'origine musulmane. Ceuxci avaient pour la plupart abandonné toute pratique religieuse, y compris dans un cadre domestique et familial, ou se limitaient à un islam très sommaire et formel. Certains n'avaient d'ailleurs reçu aucune éducation musulmane dans leur région d'origine. À cette époque, la volonté de ces Palestiniens, dont une nouvelle génération était déjà née dans l'île et dont certains avaient acquis la nationalité française, était de se fondre dans la population martiniquaise. Contrairement au fait d'être arabe et aux liens jamais coupés avec la famille restée au Proche-Orient, l'islam n'était donc, chez eux, fondateur d'aucune identité. C'est notamment par l'action énergique du marabout charismatique que l'appartenance religieuse a pu apparaître — ou réapparaître — chez eux; selon l'expression d'un Palestinien,

12. Il s'agit d'un long boubou de style africain. Ce vêtement est plus connu actuellement en Martinique qu'il ne l'était dans les années 1980.

13. On ne développera pas ici un thème important dans le processus de conversion: la perception de l'islam comme manière de renouer avec un religieux des origines.

14. Ce thème sera traité plus loin, lorsqu'il sera question de la mémoire. 
c'est grâce à fodé Marega qu'il a «repris ses sources ». Marega a « réveillé », "fait éclater» l'islam en Martinique. Son rôle a été celui d'un catalyseur, incitant les gens à pratiquer, à lire, à apprendre, à s'unir. La façon qu'il a eue d'arpenter les rues de Fort-de-France, en créant un lien entre toutes les personnes qu'il sollicitait, a pour ainsi dire constitué potentiellement dans l'espace foyalais un réseau musulman, alors que l'islam y était auparavant imperceptible, voire inexistant. L'association qu'il fonde alors ${ }^{15}$, nommée "Le lien islamique », témoigne bien de cette volonté de rassembler. De cette période, de nombreux musulmans gardent la mémoire vive. Certes, il a existé, à cette époque comme plus tard, bien d'autres voies d'islamisation ou de réislamisation. Mais ce rôle de propagateur et de rassembleur n'a jamais été contesté à Marega, même par ses pires détracteurs ultérieurs.

Ce rôle de catalyseur s'est prolongé par un véritable projet d'aménagement de l'espace (du moins à Fort-de-France) et la fondation d'une communauté. La maison du marabout est devenue le premier lieu de prière de la Martinique; Marega y tint le rôle d'imam. Il fonda un enseignement complet, créa un abattage halal et conçut, avec ses proches, le premier projet de rendre visible l'islam en Martinique en entreprenant des démarches pour la construction d'une mosquée. Une petite communauté se constitua autour de ce "guide", rassemblant des Martiniquais convertis - dont certains se revendiquent encore aujourd'hui comme ses élèves - , un groupe d'Africains et quelques Arabes palestiniens «réveillés ». Cette communauté est décrite comme véritablement utopique, marquée par le respect du fondateur, la ferveur, l'égalité entre tous les adeptes. Le modèle troeltschien ou wébérien de la secte semble particulièrement bien s'appliquer à ce cas; par ailleurs, dans le rôle de leader charismatique aménageur, ce marabout de peu renoue avec la tradition des grands chefs de confréries d'Afrique de l'Ouest.

\subsection{Deuxième étape: routinisation du charisme et problème de leadership}

Cette époque fut de courte durée. Si avec le recul du temps, certains la considèrent comme un âge d'or, pour d'autres elle représente l'âge de l'ignorance.

Plusieurs contestations sont vite apparues. La première concerna la compétence pédagogique du marabout, montrant l'insuffisance du charisme lorsqu'il se mesure au savoir. Percevant la limite des connaissances de leur guide, certains musulmans se défirent de la relation charismatique

15. Association déclarée sous la loi de 1905. 
maître/disciple pour aller étudier dans d'autres structures, en France et même en Arabie saoudite ${ }^{16}$.

Un deuxième litige eut pour objet la trop grande mobilité du marabout qui, lors de ses absences, déléguait le rôle d'imam à un de ses disciples martiniquais les plus proches. Mais la place prise par ce dernier est remise en cause par une partie des musulmans. L'héritage du fondateur (que certains commencèrent d'ailleurs à mettre en question) ne s'est pas transformé en charisme de fonction. La fréquentation de la mosquée diminua et n'est plus constituée aujourd'hui que d'un noyau de fidèles martiniquais et africains. Actuellement, cette "lignée » du fondateur est presque délégitimée, bien qu'elle existe toujours.

Par ailleurs, d'autres acteurs sont apparus, notamment un Martiniquais converti à l'islam en France dans un cadre beaucoup plus orthodoxe et qui a, en outre, fréquenté en Afrique un leader de l'islam réformiste (cheik Touré). C'est sur les thèmes du rejet du système maraboutique, de l'usage des amulettes et des pratiques de guérison que, dès 1982, il critiqua l'islam introduit en Martinique par fodé Marega, dénonçant aussi le fait que celuici se situait hors de toute hiérarchie scolaire du savoir. Le charisme du fondateur, ambivalent par nature, se retourne contre lui. On peut souligner le caractère très classique de cette condamnation du charisme maraboutique comme un "charlatanisme", reposant sur une partition non moins classique entre islam "pur» et "impur ${ }^{17}$ ».

Bientôt, le marabout fondateur fut désavoué, destitué de sa fonction d'imam. Une nouvelle association, nommée "L'entraide islamique », fut créée, ainsi qu'un nouveau lieu d'accueil.

Mais ces mêmes arguments ( «nettoyer» l'islam des pratiques maraboutiques, pallier l'incompétence) furent employés par un autre groupe mené cette fois par des Palestiniens. Entourés de certains des Martiniquais convertis et des Africains - qui, tout en soutenant leur démarche, ne manquèrent pas de les critiquer pour leur ralliement jugé bien tardif à la cause de l'islam ${ }^{18}$ - , les Palestiniens prirent l'offensive à la fois contre le marabout fondateur et son premier opposant.

16. Un très petit nombre de Martiniquais lisent et parlent l'arabe.

17. Depuis les écrits, au XIV ${ }^{e}$ siècle, du polémiste hanbalite Ibn Taymiyya, les marabouts sont accusés d' "associationnisme " (shirk), c'est-à-dire de mêler à l'islam d'autres pratiques, forgeant ainsi des innovations blâmables postérieures à l'époque prophétique (bid'a). Notons aussi que l'accusation de répandre un islam «impur» est un argument bien connu dans les luttes de pouvoir entre musulmans en Afrique de l'Ouest au XIX ${ }^{\mathrm{e}}$ siècle. À ce sujet, voir Triaud 1985.

18. Alors qu'ils étaient présents dans l'île depuis parfois 50 ans. 
À la suite de plusieurs épisodes - certains peu clairs, d'autres très durs, puisque fodé Marega faillit être expulsé de Martinique - , l'organisation de l'islam dans l'île fut très largement reprise en mains par les "Arabes ", qui par ailleurs possèdent pouvoir économique et entregent. Dans le discours des Palestiniens affleure toujours un sentiment complexe: la reconnaissance du rôle catalyseur du marabout charismatique se double du secret dépit d'avoir été devancés par un Africain, puis par des Martiniquais, alors qu'eux-mêmes, musulmans de naissance, arabisants, se pensaient investis d'une sorte de légitimité naturelle. Sur leur initiative, la situation de l'islam en Martinique se modifia considérablement. En 1984, par l'intermédiaire de relais familiaux, ils firent appel à des instances saoudiennes et sollicitèrent l'envoi d'un imam et de subventions. La Ligue islamique mondiale délègua dans l'île un imam sénégalais venant juste de terminer ses études en Arabie saoudite. Une troisième association, le Centre culturel islamique, vit le jour à Fort-de-France, ainsi qu'un troisième lieu de prière, qui aujourd'hui compte le plus grand nombre de fidèles.

L'imam sénégalais, toujours en poste actuellement, est un fonctionnaire dépendant de la Ligue islamique, payé par elle. Son savoir n'est pas contesté, et il est respecté de tous. Mais certains des acteurs de cette histoire troublée, en particulier les deux leaders martiniquais et leurs partisans, lui dénient le rôle de chef de l'ensemble des musulmans. Pour eux, il n'est que «l'homme des Arabes».

Deux traits caractérisent donc la genèse de l'islam en Martinique:

- le passage du leader charismatique à un fonctionnaire membre d'une hiérarchie, qui correspond au repliement sur un islam orthodoxe, bien qu'assez tolérant, avec une référence non négligeable à l'Arabie saoudite;

- le passage à une hiérarchie du savoir, avec le choix habile de son représentant (l'imam sénégalais), mais d'origine étrangère, qui correspond de fait à une sorte de mise à l'écart — peut-être provisoire - des musulmans martiniquais.

La Martinique compte actuellement trois lieux de prière musulmans:

- le plus ancien, tenu par l'imam martiniquais disciple du marabout fondateur; il rassemble un petit groupe assez précaire de fidèles, formé presque exclusivement de Martiniquais et d'Africains. Il est situé dans un quartier de Fort-de-France où logeaient autrefois les ruraux descendus travailler en ville, quartier très populaire actuellement habité par une population largement immigrée; 
- le second a pour animateur le musulman martiniquais qui fut parmi les premiers contestataires de l'origine maraboutique du fondateur. Ce lieu se situe à Rivière-Pilote, commune du sud de l'île où a pris naissance en 1982 le mouvement d'occupation des terres par les agriculteurs martiniquais et que C. Chivallon désigne pour cette raison comme «le haut-lieu de la résistance anti-coloniale » $(2004,401)$. Le maire de cette ville est un célèbre leader indépendantiste. Cette « orientation" protestataire est, comme on le verra plus loin, aussi décelable dans les positions défendues par les fidèles de ce lieu musulman. Ce centre, qui a vécu ces dernières années plusieurs transformations, paraît presque en sommeil. Il n'est fréquenté que par une poignée de Martiniquais, qui semblent s'orienter actuellement vers le prosélytisme itinérant et la diffusion audiovisuelle — tendance analysée plus loin;

- le troisième lieu, actuellement de loin le plus important, est celui où l'imam sénégalais évoqué plus haut dirige la prière. Ce centre regroupe des musulmans représentant l'éventail culturel le plus large: Africains, Palestiniens et autres Proche-Orientaux, Maghrébins, Comoriens de passage, Martiniquais, métropolitains convertis, etc. Il est situé dans le centre-ville de Fort-deFrance, quartier de petits commerces déclinants, que le maire actuel tente de revivifier; et dans un endroit particulier de ce centre-ville, une artère assez mal famée, proche des boîtes de nuit et de plusieurs trafics interlopes.

Il faut souligner que les deux salles de prière situées à Fort-de-France sont installées dans les étages de banals immeubles commerciaux, où l'emprise du milieu urbain se fait particulièrement sentir, y compris pendant la prière: vacarme d'un garage de réparation automobile installé au rez-dechaussée de l'un d'entre eux, bruits de la circulation, des klaxons, zouk et rap diffusés à tue-tête depuis les voitures passant dans les rues et, plus occasionnellement, tambours et cris des défilés de Carnavals...

\section{Les enjeux mémoriels}

Ces trois lieux urbains représentent une "communauté éclatée ", selon l'expression d'un Martiniquais musulman. Leur séparation relève, certes, de clivages politiques et stratégiques, ainsi que d'un problème de leadership. Mais on peut déceler, derrière ces raisons historiques, des enjeux symboliques très 
forts, qui reposent sur des mémoires et des élaborations identitaires personnelles et collectives très différenciées.

Le sentiment de l'umma, la communauté des croyants, censée souder entre eux tous les musulmans, s'énonce avec force chez tous les musulmans de Martinique, toutes origines confondues; par elle s'expriment la solidarité, l'égalité de tous qui transcendent les particularismes. Mais il apparaît bien vite que cette affirmation de l'umma, qui se réfère au présent, ne parvient pas toujours à masquer les appartenances. Notons d'ailleurs - effet d'un universalisme qu'on pourrait dire polarisé - la marque de l'Arabie saoudite dans l'organisation de cette umma: c'est elle qui fournit, en Martinique, la très grande majorité des livres rédigés en français et distribués gratuitement, dans lesquels la plupart apprennent l'islam; c'est aux émissions religieuses saoudiennes qu'ont accès ceux qui ont fait l'acquisition d'antennes paraboliques; et c'est, la plupart du temps, aux décisions saoudiennes qu'on se réfère pour déterminer la date ou l'heure des fêtes ${ }^{19}$. Dans la vie musulmane martiniquaise, malgré les rassemblements à l'occasion de la prière du vendredi ou des fêtes, malgré des échanges plus denses lors du mois de ramadan et de très rares événements collectifs ${ }^{20}$ (un piquenique musulman en 2004), évoquer l'umma est l'expression d'un idéal ou d'une norme lointains. Comme le dit très clairement un musulman martiniquais: «l'islam est là pour transcender les clivages tribaux; mais une fois la prière finie, chacun retourne dans sa communauté qui n'est pas l'umma: les différences ne sont pas transcendées".

Je vais montrer en quoi des mémoires différentes qui se rencontrent ou même s'affrontent font de l'umma une utopie quelque peu lointaine et comment l'invention tâtonnante et progressive de la vie musulmane en Martinique se nourrit d'initiatives où sont à l'œuvre ces mémoires.

\subsection{Le projet de construction d'une vraie mosquée}

On l'a vu, le projet de construction d'une mosquée avait été formulé par le marabout charismatique; il a été repris par le groupe actuellement dominant. Tous n'y ont pas adhéré, notamment les gens de passage, qui se satisfont des salles de prière dans des lieux anonymes, et qui évitent tout investissement financier trop important. Une autre réserve est venue de

19. Alors que d'autres références sont possibles, ce que ne manquent pas de faire remarquer certains musulmans, africains notamment.

20. Qui ne réunissent d'ailleurs jamais les trois groupes. 
ceux pour lesquels l'intention de donner, autant qu'un lieu de culte satisfaisant, une visibilité aux musulmans de Martinique — phénomène très récent - semblait prématurée ${ }^{21}$, ou trop prétentieuse ${ }^{22}$. Mais selon l'opinion majoritaire, cette construction et les activités qu'elle entraînerait pourraient dynamiser les musulmans. Le projet d'une grande mosquée, conçue pour les générations futures, qui donnerait une image à l'islam en Martinique et serait un phare de l'islam dans toute la Caraïbe francophone l'a emporté. Il s'agirait de la première inscription réelle de l'islam dans l'espace et dans le futur martiniquais. Après les multiples péripéties de l'achat d'un terrain, un petit groupe de musulmans a fait le choix d'une architecture de style oriental avec minaret, ce qui rendrait le bâtiment clairement identifiable ${ }^{23}$; les plans en ont été commandés à un architecte de Fort-deFrance. Or, comme on l'a déjà souligné, le pouvoir économique appartient aux Palestiniens, par lesquels passent les liens avec l'Arabie saoudite qui finance partiellement la construction des mosquées. L'un d'entre eux, le trésorier financeur, a de fait écarté l'architecte martiniquais et imposé un projet concurrent plus monumental: la reproduction d'une mosquée de Ramallah, sa ville natale. Cette transformation a impliqué, au fil du temps, de nombreuses complications dans la construction ${ }^{24}$, aucun entrepreneur martiniquais ne se déclarant compétent pour construire certains arcs, dont les moules ont dû être réalisés par des maçons égyptiens, le chantier étant organisé pendant un temps par un Libanais de Paris... Ce choix architectural traduit la prégnance très forte d'une mémoire particulière, celle des origines palestiniennes, imposée par un petit groupe (et spécialement une famille puissante) à l'ensemble des musulmans martiniquais, et qui marquera l'espace. Certes, tous les musulmans de l'île n'ont pas attribué à cette décision la valeur d'une véritable emprise, certains ne soulevant aucune objection, d'autres réprouvant plutôt son gigantisme ou son coût. Mais les critiques les plus acrimonieuses ont été formulées par certains Antillais: pour eux, il s'agissait d'une part de la confiscation par les «Arabes » d'un projet formulé naguère par fodé Marega et auquel ils étaient associés, et d'autre part d'une marque trop exclusive de la culture arabe sur ce projet

21. Le temps de "s'exhiber» n'était pas encore venu, en particulier à cause de la scission en trois groupes.

22. Ce que certains critiquaient était le luxe et le coût de la construction projetée.

23. On ne peut pas évoquer ici les multiples tractations et rétractations qui eurent lieu entre les musulmans, les voisins, la municipalité.

24. Commencée en 1993, mais interrompue plusieurs fois par manque de crédits. L'infrastructure était en voie d'achèvement en 2006. 
et en Martinique; prolongeant cette critique, un petit nombre va jusqu'à formuler la crainte du wahhabisme.

Sans développer ici tous les éléments montrant l'existence d'une mémoire arabe, on peut simplement mentionner: l'usage de l'arabe dialectal que les Palestiniens parlent entre eux, ce qui les isole des autres musulmans; les réunions informelles sous un arbre face à la mosquée ${ }^{25}$, après la prière du vendredi, ce que ne manquent pas de souligner certains musulmans antillais; le manque de participation des femmes "arabes » à la plupart des événements de convivialité collective, etc.

Un autre élément relatif à l'impact de mémoire de groupes particuliers sur la vie de l'islam en Martinique est la célébration du Magal. Cette fête particulière aux mourides ${ }^{26}$ du Sénégal commémore le départ en exil du fondateur de la confrérie, cheikh Amadou Bamba. Elle n'est pas considérée comme une fête concernant l'ensemble des musulmans; ainsi, au Sénégal, aucun jour de congé n'est attribué systématiquement pour sa célébration. En Martinique vivent quelques familles mourides, en nombre variable selon les périodes. Parmi elles, celle de l'imam du plus grand centre de prière: il est membre d'une famille très réputée et respectée, celle de l'actuel khalife général des mourides ${ }^{27}$, bien que lui-même, ayant fait ses études en Arabie saoudite, soit très éloigné des pratiques maraboutiques actuelles. Les mourides de Martinique se réunissent entre eux pour des séances de prière particulières ${ }^{28}$. Pendant le temps où ils étaient assez nombreux, ils ont fait fonctionner une caisse de solidarité spécifique, et ils célébraient entre eux le Magal. Récemment, cette fête africaine et mouride a eu lieu au sein de la mosquée du plus grand des lieux de prière. L'imam a expliqué aux fidèles rassemblés l'histoire de cheikh Amadou Bamba, sans insister sur la spécificité mouride, mais en accentuant au contraire l'universalité de la fête. Quelques Palestiniens y ont participé, tant pour manifester de façon volontariste leur appartenance à l'umma que pour témoigner de leur respect envers l'imam et pour éviter de faire du « racisme » (c'est le terme qui fut employé, qui semble indiquer le sentiment complexe de crainte, mais aussi de latence des particularismes). Ont aussi pris part à la fête des Africains issus de tous horizons musulmans et de toutes nationalités présentes en Martinique. Ce fait peut s'interpréter comme un élargissement classique du cercle des appartenances en émigration,

25. Rappelons que les musulmans palestiniens fréquentent exclusivement la salle de prière la plus importante de Fort-de-France.

26. Il s'agit d'une confrérie soufie très vivace au Sénégal.

27. La famille Mbacké, qui est celle du fondateur de la confrérie.

28. La récitation de khassaïd, poèmes religieux écrits par cheikh Amadou Bamba. 
mais aussi par la réputation de l'imam comme Africain, indépendamment de son appartenance confrérique. Retraçant l'histoire de l'islam dans l'île, nous avions mentionné l'origine malienne du marabout fondateur : ce fait a beaucoup joué dans le regroupement des Africains autour de lui. Après son départ de l'île, c'est en partie par fidélité au maître que nombre de musulmans sont restés groupés autour de son disciple martiniquais. Mais dès la venue de l'imam sénégalais, un grand nombre d'Africains ont rejoint ce dernier, tant en raison du savoir qui lui était reconnu que par souci de se rallier à un Africain, dont par ailleurs la famille et le nom étaient renommés.

Si la mémoire palestinienne contribue à modeler la structuration spatiale de l'islam martiniquais, la mémoire africaine en modèle donc les rituels. On peut en trouver d'autres signes: la convivialité des fêtes, et notamment les repas collectifs, la cuisine, sont pratiquement toujours majoritairement pris en charge par les Africains. Ces fêtes sont d'ailleurs pour plusieurs d'entre eux des temps de nostalgie, où chacun évoque la façon dont tel épisode, tel geste se déroulent chez lui; sans doute est-ce parce que la mémoire du pays est beaucoup plus vive chez eux qu'elle ne l'est pour les Palestiniens dont l'émigration est plus ancienne ${ }^{29}$. Par ailleurs, il existe en Martinique plusieurs associations d'originaires d'Afrique (Sénégalais, Guinéens, etc.). Il faut toutefois remarquer que l'introduction du Magal dans le «corpus» des fêtes musulmanes martiniquaises, de même que la marque de l'Afrique dans la convivialité sont sporadiques et précaires: elles dépendent de la présence dans l'île d'un nombre suffisant de fidèles originaires de ces pays; or, une bonne partie d'entre eux se déplace avec une grande fréquence, parfois sans retour en Martinique. Cette différence du type de mobilité entre Palestiniens, plus sédentarisés, et Africains influe sur la nature et l'impact de leurs mémoires respectives.

Mais reprenons la célébration du Magal: des musulmans martiniquais y participent également, certains sans savoir qu'il s'agit d'une fête mouride, d'autres parce qu'ils s'en remettent à l'imam qui les forme, d'autres enfin en raison d'un rapport très particulier qu'entretiennent les Martiniquais avec l'Afrique. Il est difficile de résumer sans schématiser les sentiments très complexes et contradictoires que les Martiniquais entretiennent avec l'Afrique et les Africains. On peut cependant discerner quelques lignes de force ${ }^{30}$.

29. Même si, comme on l'a noté, les liens avec le pays d'origine sont largement maintenus.

30. Notre objectif ici n'est en rien de synthétiser l'histoire des rapports entre Africains et Martiniquais, mais de rester au plus près de nos données de terrain recueillies en milieu musulman en Martinique. 
Des liens entre Martiniquais et Africains sont et ont été tissés, en métropole comme en Martinique, et nous avions souligné qu'ils constituaient pour les Martiniquais l'une des voies de conversion à l'islam. Le point d'accroche de ces liens est très souvent l'admiration pour la convivialité des Africains (en wolof, teranga, " hospitalité ", élevée, il est vrai, au rang d'art de vivre et de morale sociale $\left.{ }^{31}\right)$. Mais il s'agit souvent de relations nouées dans la contemporanéité, le hic et nunc, sans référence au passé. Se sentir proche d'un Africain ne veut pas nécessairement dire qu'on accepte l'Afrique.

Car le rapport à l'Afrique reste un des points focaux du rapport difficile au passé. Si aujourd'hui encore certains des Martiniquais musulmans détestent l'Afrique, rejettent violemment tout lien avec ce continent ou pratiquent l'ignorance et la méconnaissance, c'est que l'évoquer constitue un rappel implicite de l'esclavage; aborder de front ce thème se résume souvent à une accusation sans appel: les Africains auraient vendu leurs propres frères ${ }^{32}$. Ainsi, certains Martiniquais se démarquent très nettement des Africains, refusant par exemple de leur louer des logements. Cette attitude de rejet s'explique par la méfiance perceptible en Martinique à l'égard de tout étranger, mais surtout par le maintien du désir, poursuivi bien longtemps, de se niveler à la métropole. Cette distance à l'égard de l'Afrique n'empêche nullement qu'en Martinique tout Africain soit réputé avoir une force particulière, détenir des secrets : évité pour les raisons exposées plus haut, il est en même temps recherché et craint. D'où le succès, dans le domaine magico-religieux, des marabouts, mais chacun a, à ce sujet, son anecdote personnelle: tel passe pour attirer les clients du fait même de sa présence dans telle entreprise, tel ne ferme jamais sa maison sans crainte des cambriolages, etc. Ce supposé pouvoir de l'autre, particulièrement puissant et redouté en ce qui concerne l'Afrique, peut être interprété comme un des effets de la mise à distance. Mais on voit l'entremêlement de forclusion, de répulsion et d'attirance dans cette relation avec l'Afrique: la mémoire collective antillaise est à ce sujet très ambivalente.

Cependant, hors de la question des origines, les relations entre Antillais et Africains sont assez anciennes: on sait que de nombreux Antillais ont été

31. C'est d'ailleurs le nom d'un restaurant africain très connu de Fort-de-France.

32. Accusation qui semble sous-entendre que les Africains contemporains en portent leur part de responsabilité, et qui globalise l'ensemble des Africains, musulmans ou non. Sur ce sujet très controversé, voir la récente synthèse de Pétré-Grenouilleau (2004, 444-457). Dans un séminaire tenu autour de cet ouvrage en mai 2005 dans le cadre de l'Université Paris 1, l'historien de l'Afrique J.-L.Triaud estimait que le facteur islamique dans la traite ne devait pas être surestimé. 
cadres, enseignants en Afrique dans l'administration coloniale; il en résulte un sentiment mêlé de supériorité et d'une solidarité fondamentale, de telle sorte qu'actuellement, certains Antillais issus de ces familles ne vivent qu'en milieu africain ou s'en sentent très proches. Une association ancienne (Afrique-Martinique), aujourd'hui disparue ${ }^{33}$, montre les affinités culturelles et une proximité de fait toujours attestées, comme en témoigne l'existence de plusieurs groupes récents ${ }^{34}$. En outre, plusieurs Africains sont mariés à des Martiniquaises.

À ces mémoires ambiguës, qu'il est impossible de réduire à une position univoque, il faut bien sûr associer, à l'opposé du rejet, le poids de tout le courant bien connu né de la théorie de la négritude et de la recherche des racines pour en finir avec l'éducation coloniale. Ainsi, avant même la rencontre avec son maître, l'imam martiniquais d'un des centres de prière, disciple très proche du marabout fondateur, a fait plusieurs voyages en Afrique où il a vécu dans des familles; pour lui, l'Afrique est "son origine » revendiquée, que son attachement pour son maître n'a fait que renforcer. Le même lien existe pour le leader du centre de Rivière-Pilote, même si ses références sont différentes. On est donc là dans une mémoire longue et non dans l'immédiateté de la relation. Mais si nombreux sont les musulmans antillais qui s'intéressent à l'Afrique, font le voyage vers Dakar, sont attirés par les mystères imaginés de Touba, berceau du mouridisme, par les paysages africains, par les personnages religieux vénérés..., cette curiosité s'ancre aussi dans des intérêts présents, et le souci des racines n'en est pas la seule explication. Ce continent exerce sur eux une sorte de fascination, ce qui n'est pas du tout le cas pour l'univers culturel arabo-musulman. Pour autant - exacte réciproque de ce que nous avancions plus haut être attiré par l'Afrique ne signifie pas nécessairement accepter l'Africain, ce qui montre bien l'extrême complexité de ces relations. Mais il importe de noter qu'entre les deux grandes positions idéologiques antagonistes le rejet de l'Afrique et l'affirmation de la négritude - que les musulmans martiniquais ont partagées et partagent encore comme les autres, se tisse et s'immisce une multitude d'attaches et de petits liens latéraux, s'élaborent des bribes de récits et de représentations plus souterraines et particulières, qui mériteraient à eux seuls une étude.

33. Elle existait à l'époque du marabout fondateur.

34. Telle l'association martinico-sénégalaise AK, dont le nom dénote la liaison en créole et en wolof. 
Que ce soit dans le déni ou l'affirmation, dans une mémoire longue ou plus courte, mais toujours dans un dialogue difficile et passionnel, pour nombreux musulmans martiniquais, l'espace de l'islam ne se limite donc pas à leur île, mais se déploie, de façon réelle ou imaginaire, vers l'Afrique. On le voit dans les fêtes de la plus grande des salles de prière, on le voit aussi dans le souhait de l'imam martiniquais disciple du marabout fondateur de créer une vie communautaire très marquée par l'Afrique, et dans bien d'autres détails qu'il est impossible à développer ici ${ }^{35}$.

Mais en dehors de ce rapport à l'Afrique, les musulmans martiniquais établissent et composent d'autres liens entre mémoire et territoire caribéen.

$\mathrm{Si}$, il y a quelques années, un archéologue métropolitain quelque peu naif en poste en Martinique pouvait s'étonner que les Martiniquais s'intéressent aux périodes précolombiennes, mais non aux époques ultérieures (celles de l'esclavage - époques forcloses), cette réalité semble s'être largement modifiée aujourd'hui: depuis une dizaine d'années, et plus particulièrement depuis la célébration du $150^{\mathrm{e}}$ anniversaire de l'abolition de l'esclavage (1998), le sujet commence à être abordé pas uniquement dans les cercles intellectuels. Cette résurgence de la mémoire de l'esclavage est aussi le fait des musulmans.

On connaît la thèse longtemps défendue notamment par E. Glissant, avant qu'il ne revienne sur ses propres interprétations. Il parle de mémoire raturée, de biffure : la langue, les dieux, les coutumes, les outils se seraient perdus sur les bateaux négriers et la seule mémoire possible ne fonctionnerait que par traces. Le travail de plusieurs ethnologues, historiens et géographes montrant que s'il n'existe pas de grand récit communautaire globalisant, on trouve une multitude de petits récits ou d'expériences qui dessinent autant de chapitres du passé martiniquais ${ }^{36}$, n'empêche pas que cette thèse du vide de la mémoire et de la transmission soit encore formulée. Voici par exemple ce que dit un musulman martiniquais: "aux Antilles, on est un peuple d'esclaves qui n'a pas d'origine, ne peut pas remonter loin

35. Tel le port de certains vêtements directement inspirés de l'Afrique.

36. Voir notamment Jolivet 1987, qui montre l'évolution d'une mémoire historique passant du schoelcherisme au marronnisme, et Price 2000, qui met en exergue comment la vie de résistance d'un personnage original condamné au bagne nourrit la mémoire fragmentaire, multiforme et contradictoire des Martiniquais. Voir aussi Chivallon 1998, qui souligne que l'appropriation des terres des mornes par les esclaves libérés dans la période post-esclavagiste a constitué, non par une mise en récit, mais “en actes”, l'émergence d'une manière d'exister autrement que par la vie dans les plantations (237). 
dans sa généalogie; un peuple de misère qui est condamné au travail pénible, qui n'a pas de temps pour la religion, qui n'a pas vraiment d'histoire. Les parents n'ont raconté que la misère qu'ils ont vécue, mais pas les ancêtres ". Sans qu'il s'agisse en rien de réaffirmer le thème de l'aliénation, l'islam est une des façons que certains Martiniquais ont choisies pour pallier le manque de profondeur de la mémoire familiale, pour remplir l'absence de récit et le vide: cette absence donne sa force à l'idée d'un homme universel (al-insân en arabe) postulé par l'islam, où chacun est tout simplement mais rien de moins que fils d'Adam. Le vide de la mémoire devient un plein musulman/universel, et l'Antillais se fond dans l'universel. D'où des affirmations telle celle-ci: "dans l'islam, on n'a pas d'identité, tout le monde est pareil »; ou encore: «l'islam, c'est une nouvelle identité, le nom musulman suffit ». Le ciment de l'être antillais serait l'universel de l'islam, notion métaphysique se situant au-delà d'une ancestralité historique.

Cet usage de l'islam a une variante, où il ne s'agit plus d'oubli et d'absence, mais bien de mémoire, celle de l'esclavage. Cet homme universel de l'islam est celui qui transcende les races et les classes: il n'y a plus ni père ni mère, il n'y a que des frères. Pour les Martiniquais, l'inscription dans l'islam efface l'oppression, les inégalités et les duretés de l'esclavage, contrairement à la religion imposée aux esclaves, le catholicisme, dont les Antillais ont pu mesurer les effets inégalitaires jusqu'à le délégitimer. Audelà des particularismes et des clivages, "l'islam rattache les Antillais à l'histoire humaine, permet de rebâtir la société martiniquaise sans race, de se rattacher à l'humanité dans son ensemble. Le combat n'est pas de revendiquer sa couleur, mais d'être un homme». On voit, au passage, que les musulmans martiniquais se détachent de la position des Black Muslims nord-américains : pour eux, affirmer la couleur noire des prophètes n'a pas de sens et se révèle "puéril » : rien d'autre que «du racisme à rebours ${ }^{37}$ » .

On peut dire de ces deux variantes de l'universel qu'elles ancrent les musulmans martiniquais dans un espace soit utopique, soit planétaire, dont ils seraient une partie. Et l'on discerne bien l'interprétation particulièrement forte de la notion de l'umma en milieu antillais.

Mais une autre voie est aussi empruntée par d'autres musulmans. Ceuxci combinent la légitimation de l'islam dans sa dimension universelle avec l'affirmation d'une présence ancienne de l'islam dans la Caraïbe, dont ils cherchent et dont ils pensent trouver les signes: la tête couverte des femmes

37. Toutes les expressions entre guillemets émanent de musulmans martiniquais. 
antillaises qui correspondrait au voile musulman, la façon de laver la viande, et jusqu'au vêtement des Amérindiens qui serait proche de celui des musulmans. Cette certitude qu'il y avait parmi les esclaves nombre de musulmans, que les esclaves étaient même en majorité musulmans, certains disent en avoir des preuves. La fiction de la filiation possible (même si on ne va pas vraiment jusqu'à la démontrer) paraît être partiellement entretenue par la lecture du fameux ouvrage Roots, d'Alex Haley (1976), que les musulmans martiniquais connaissent. À l'appui de cette affirmation de la présence de musulmans parmi les esclaves, l'un d'eux évoque la découverte récente en Guadeloupe d'un cimetière d'esclaves (voir Chivallon 2002, 604609). On ne sait, certes, rien sur la présence historique de musulmans parmi ces esclaves : c'est une hypothèse implicite que font ceux qui tirent argument de l'existence de ce lieu. Mais qu'historiquement on ait peu de traces n'est pas ici la question.

Un pas de plus est franchi dans cette recomposition mémorielle de l'ancrage ancien de l'islam dans la Caraïbe lorsque sont considérés comme des musulmans les héros de la révolte des esclaves en Haïti ${ }^{38}$ : tel Makandal, esclave haïtien dont on dit qu'il lisait de droite à gauche, comme se lit l'arabe; tel Boukman, dont le nom signifierait «l'homme du livre », ce livre étant le Coran. De même, le nom du lieu d'où serait partie la révolte, le Bois-Caïman, serait en fait Kaye-Imam ( «la maison de l'imam»). Il en est de même pour la réappropriation musulmane des nègres marrons. La seule librairie musulmane qui ait existé dans l'île se nommait "Librairie tiers-mondiste Makandal ${ }^{39}$ » : l'intention est claire. L'homme qui l'a créée, aujourd'hui à la tête du centre de Rivière-Pilote, est l'auteur d'un manuscrit non publié, fruit de ses recherches militantes pendant de longues années, intitulé «Les musulmans pré- et post-colombiens. Biographie islamique de Malcolm X». Il faut noter que ces héros, revendiqués comme les leurs par une partie des musulmans antillais, le sont aussi par les adeptes du vaudou.

Enfin, le dernier pas est d'attribuer la découverte de la Caraïbe non à Christophe Colomb, mais à des musulmans africains ou maures, découverte dont la mémoire se serait perdue ou aurait été occultée. Ce thème de l'occultation a d'ailleurs une grande puissance explicative: de même qu'aurait

38. La réappropriation des héros haïtiens n'est en rien un hasard: d'une part, comme le souligne Jolivet (1987), l'histoire de la Martinique est dépourvue de héros libérateur auquel les musulmans, comme d'autres Martiniquais, pourraient s'identifier; d'autre part, la libération de Saint-Domingue (1803) est bien antérieure à celle de la Martinique et de la Guadeloupe.

39. D'abord établie à Fort-de-France, puis à Rivière-Pilote, elle semble avoir formellement disparu (constatation faite lors de mes dernières enquêtes de terrain en 2004). 
été cachée l'ancienneté de la présence de l'islam dans la Caraïbe, de même on aurait dissimulé la prophétie du prophète Mohamed, annoncée dans un texte que l'orthodoxie chrétienne et la quasi-totalité des critiques considèrent comme apocryphe (l'évangile selon Barnabé); prophétie encore plus occultée dans les Antilles où les colons tenaient les esclaves dans l'ignorance. La particularité de cette affirmation mémorielle relative à une découverte de la Caraïbe par des musulmans antérieurement à Christophe Colomb est qu'elle permet de reconstituer une identité musulmane caraïbe antérieure à l'esclavage, c'est-à-dire antérieure à la domination.

Ces arguments circulent de bouche à oreille et sont retravaillés, comme toute transmission orale. Mais l'une des origines possibles me semble être le livre d'un journaliste trinidadien converti à l'islam, Andy Thomas, qui a pris symboliquement le nom d'Abdullah Bilal ${ }^{40}$ Omowalé. Cet ouvrage intitulé L'islam, âme de l'bumanité (2000), qui cherche notamment à revaloriser l'histoire des musulmans d'Afrique noire, souligne que ceux-ci, établis "aux Caraïbes et en Amérique du Sud » à l'époque précolombienne, y jouèrent un grand rôle dans les mouvements de résistance (109). Disponible dans toutes les librairies musulmanes de métropole et constamment réimprimé depuis sa parution en 1991, ce livre est également connu et commenté en Martinique ${ }^{41}$.

On voit donc que sous des registres différents, il s'agit de construire l'espace caraïbe comme un espace musulman de longue date: soit par défaut de mémoire avec l'usage de l'homme universel défini par l'islam, qui inclut l'espace caraïbe tout en le dissolvant, soit par une inscription réelle et une mémoire presque glorieuse et combattante. En somme, il s'agit de montrer que l'islam a sa place dans la mémoire héroïque de la Caraïbe et dans la lutte contre l'esclavage.

Si les musulmans d'origine martiniquaise ne sont pas les seuls à légitimer ainsi leur existence — car ces arguments sont repris, de façon peut-être moins insistante, par des musulmans d'autres origines, en particulier africains - , le bénéfice pour eux en est évident. Il s'agit de reconstituer une fierté antillaise. Le leader du centre de Rivière-Pilote va même jusqu'à affirmer qu'après une première étape positive, celle de la reconnaissance de l'esclavage comme crime contre l'humanité, il s'agit maintenant de valoriser la culture islamo-caribéenne comme patrimoine caribéen.

40. Bilal était le nom de l'esclave éthiopien du Prophète. Ce prénom a été aussi choisi par plusieurs Martiniquais lors de leur conversion à l'islam, et il est fréquent chez les Africains-américains musulmans aux États-Unis.

41. Il faisait partie du fonds de la Librairie Makandal, et c'est par ce canal que plusieurs musulmans martiniquais et d'autres l'ont lu. 
Dans la période actuelle, avec l'émergence du thème de l'esclavage, on assiste à une revalorisation des éléments culturels martiniquais : musiques de percussion (qui auparavant rappelaient trop l'Afrique), chanté Nwel, éclat des festivités du Carnaval, création en 1992 de l'Écomusée de Martinique qui retrace la vie dans l'île depuis les périodes amérindiennes jusqu'à l'époque contemporaine. Ce sont les rastas qui ont montré le chemin, Bob Marley (et son combat pour la dignité) étant pour certains le premier héros positif de l'histoire martiniquaise. Il apparaît clairement que, avec leurs propres arguments, certains musulmans martiniquais commencent à s'intégrer à ce mouvement.

De façon interne aux groupes musulmans de Martinique, la territorialisation de l'islam dans la Caraïbe et la mémoire de son ancienneté glorieuse ont plusieurs conséquences.

D'abord, elles remettent en question le leadership des «Arabes » en particulier, que le leader de Rivière-Pilote accuse, de façon un peu outrancière, de vouloir "déconnecter l'islam en Martinique de ses racines ", sa critique à l'encontre des Africains étant plus ambivalente. Ce qui est vécu de façon forte comme une domination s'exprime en des termes dépourvus de toute ambiguité : «ils nous considèrent comme leurs esclaves ", "ils se prennent pour nos maîtres ». Or, les arguments qui viennent d'être évoqués tendent à postuler qu'aucune autorité étrangère n'est vraiment légitime. Cependant, on peut se demander si la contestation, voire le refus de tout encadrement étranger ne traduit pas la fragilité des Martiniquais et la trace de blessures non refermées.

Autre conséquence : le scrupule des musulmans martiniquais à respecter l'umma en raison de la force que représente pour eux l'idée de l'universalité de l'homme qu'elle recèle - attachement à l'universalité qui s'explique aussi parce que, dans leur île, les Martiniquais ne sont pas en situation minoritaire - commence à se fissurer : certains musulmans martiniquais émettent l'idée qu'ils peuvent avoir entre eux des intérêts spécifiques, qu'ils peuvent légitimement s'organiser sans toujours subir les projets des autres. Mais il s'agit là de velléités encore très timides que sont loin de partager tous les musulmans martiniquais, d'autres concevant toute particularisation comme un repli et un enfermement. En outre, refuser le leadership des autres ne conduit pas nécessairement les Martiniquais musulmans à s'entendre entre eux - ce que le leader de Rivière-Pilote déplore comme une trace de l'esclavage, du "vieux combat nèg cont' nèg ».

Dernière conséquence, enfin, de cette affirmation d'une mémoire historique «islamo-antillaise»: se définir non plus comme simples musulmans, 
mais comme musulmans martiniquais ne change rien au désir d'œuvrer dans le sens de l'islam, partout et pour tous; mais il s'agit désormais pour ces fidèles d'affermir et de faire valoir leur participation et leurs initiatives ${ }^{42}$. Le leader de Rivière-Pilote a un cheminement plus idéologique et plus radical: son but est de montrer que l'islam en Martinique n'est pas «l'arabisme ». Sans quitter l'universalité, il poursuit le but de développer la culture locale. Sa mosquée de Rivière-Pilote est éloignée des grandes villes pour pouvoir toucher les populations des campagnes. Il insiste sur le fait que ses bâtiments sont construits dans le bois et le style du pays. Il parle aux gens en créole, il sillonne les campagnes ${ }^{43}$ dans l'espoir de fonder, petit à petit, un groupe musulman au contact profond avec la population. Cette sensibilité martiniquaise militante très forte semble cependant parfois à la limite du repli identitaire, et fait taxer cet homme d' " islamo-nationaliste » par ses détracteurs. Cependant, sa position a peu à voir avec les revendications politiques des Africains-américains; et, de façon très paradoxale, c'est un certain islam de France qui constitue un de ses modèles; n'oublions pas que la Martinique est un département français.

Le parcours de cet homme représente certainement le point extrême de la revendication née de l'expression d'une mémoire musulmane dans la Caraibe. Mais il reste à observer, dans la durée, si ses positions resteront peu suivies ou si elles finiront par travailler celles des musulmans martiniquais les plus modérés et les plus attachés à l'universalisme, jusqu'à les engager à imprimer plus fortement leur marque sur l'islam dans l'île. Car si la mémoire palestinienne tend à trouver son expression principale dans le façonnement de l'espace musulman martiniquais, si celle des Africains se polarise sur les rituels, on peut avancer qu'à travers l'élaboration d'une mémoire qui leur est propre, les Martiniquais musulmans cherchent et affirment leur légitimité et leur place dans cet ensemble ${ }^{44}$.

42. Ce qui semble plus difficile dans le centre le plus important de Fort-de-France, où la population est plus mêlée et où les investissements financiers sont plus importants.

43. Mode de fonctionnement qu'il a choisi en constatant que son centre attirait peu des musulmans très dispersés et en considérant que sa tâche était plutôt de faire connaître l'islam. Cet homme assure également une émission hebdomadaire d'une heure consacrée à l'islam sur une des nombreuses radios de la bande FM martiniquaise.

44. Il s'agit bien sûr, ici, de schématiser de grandes tendances, la réalité étant faite de toute une gamme de collaborations effectives et de positions nuancées. 


\section{Conclusion}

La présence de l'islam en Martinique est récente; cette raison, à laquelle s'ajoute la mobilité de sa population, explique que ses lieux, ses rituels, ses modes d'organisation et ses références soient loin d'être fixés. Mais ce qui ressort, c'est combien cet espace musulman en élaboration et son ancrage sur le sol martiniquais sont pris dans une double contrainte: d'une part, fonder et assurer collectivement l'islam en Martinique - à la fois pour cimenter un groupe en affirmant son unicité, son universalité, et pour attester son existence vis-à-vis de l'extérieur, dans un lieu où il est fortement minoritaire - ; d'autre part, de façon consciente ou plus ou moins latente, s'agissant de musulmans d'origines très diverses, laisser filtrer quelque chose de ces origines, souci dont l'importance est tout aussi grande pour les Martiniquais que pour les autres. Ces mémoires particulières entrent en conflit.

Sont en jeu dans cette double contrainte plusieurs types d'espaces: celui, très matérialisé, de la Martinique, qui reste concrètement à aménager ; les réseaux translocaux (Afrique, métropole, Proche-Orient) dus à la migration et à des liens anciens et complexes; l'espace global de la Caraïbe, objet pour les Martiniquais d'élaborations d'une mémoire historique d'autant plus fertile que les traces historiques ou archéologiques sont peu nombreuses; enfin, l'espace utopique de l'universalité. Peut-être pourra-t-on dire un jour que l'islam martiniquais est fait de tout ce que la mémoire de ces lieux différents implique. L'intérêt de cet exemple martiniquais est de révéler les choses en train de se faire. Mais il montre aussi que la multiplication conflictuelle de ces espaces et de ces mémoires n'est qu'un signe de plus de la difficulté des constructions identitaires dans les Antilles françaises, difficulté que l'évocation contemporaine parfois post-moderne du «métissage» ne paraît en rien régler.

\section{Références}

Chivallon, C. (1998), Espace et identité à la Martinique. Paysannerie des mornes et reconquête collective. 1840-1960, Paris, CNRS.

(2002), "Mémoires antillaises de l'esclavage ", Ethnologie française, 37/4, p. 601-612.

(2004), «Espace, mémoire et identité à la Martinique. La belle histoire de "Providence" ", Annales de géographie, 638-639, p. 400-424. 
Cuche, D. (2001), "Un siècle d'immigration palestinienne au Pérou. La construction d'une identité spécifique", Revue Européenne des Migrations Internationales, 17/3, p. 87-118.

Haley, Alex (1976), Roots: The Saga of an American Family, Garden City, Doubleday.

Hurbon, L. (2000a), "Les nouveaux mouvements religieux dans la Caraïbe", dans L. Hurbon, dir., Le phénomène religieux dans la Caraibe, Paris, Karthala, p. 307-354. (2000b), "Pentecôtisme et transnationalisation dans la Caraïbe ", dans J.P. BASTIAN, F. CHAMPION et K. RousSELET, dir., La globalisation $d u$ religieux, Paris, L'Harmattan, p. 125-138.

Jolivet, M.-J. (1987), «La construction d'une mémoire collective à la Martinique: du schoelcherisme au marronnisme », Cahiers d'études africaines, 107-108, p. 287-309.

Massé, R. (1978), Les Adventistes du septième jour aux Antilles françaises, Montréal, Publications du Centre de recherches caraïbes.

Massé, R. et V. Poulin (2000), «La place des Églises fondamentalistes dans la société et dans la culture martiniquaise ", dans J. BERnABé, J.-L. BONNIOL, R. CONFIANT et G. L'ÉTANG, dir., Au visiteur lumineux. Des îles créoles aux sociétés plurielles. Mélanges offerts à Jean Benoist, PetitBourg, Ibis rouge, p. 403-415.

Omowalé, A.B. (2000) [1991], L'islam, âme de l'humanité, Strasbourg, Maison de la Sagesse.

Pétré-Grenouilleau, O. (2004), Les traites négrières. Essai d'histoire globale, Paris, Gallimard (Bibliothèque des Histoires).

Planson, S. (2005), "Démographie. Une croissance démographique au ralenti ", AntianeEco, 64 (octobre), accessible en ligne à l'adresse : $<w w w . i n s e e . f r / f r / i n s e e \_r e g i o n s / g u a d e l o u p e / p u b l i / A E 64 m a . h t m>$.

Price, R. (2000), Le bagnard et le colonel, Paris, Presses universitaires de France (Ethnologies).

TRIAUD, J.-L. (1985), «Le renversement du souverain injuste. Un débat sur les fondements de la légitimité islamique en Afrique noire au XIX ${ }^{\mathrm{e}}$ siècle ", Annales ESC, 3, p. 509-519. 


\section{Résumé}

En Martinique, l'islam est apparu dans les années 1980. Représenté d'abord par des migrants venus d'Afrique de l'Ouest et du Maghreb, il a gagné progressivement des Martiniquais qui se sont convertis tandis que d'autres, notamment des commerçants palestiniens présents dans les îles depuis longtemps, reprenaient une pratique religieuse qu'ils avaient abandonnée. La question de l'origine et la mémoire des lieux jouent un rôle essentiel dans les essais de structuration de l'islam dans les îles et dans la séparation en différents groupes. On identifiera trois références antagonistes, variables dans leur efficacité et leurs effets, qui orientent de façon différente selon les groupes tant l'agencement des lieux que les rituels et les comportements des fidèles : la référence à l'arabité et à un terroir « originel » oriental; la référence (ambiguë) à l'Afrique; la référence au terroir antillais.

\section{Abstract}

Islam appeared in Martinique during the 1980's, due to the appearance of migrants coming from West Africa and Maghreb. Certain natives from Martinique then converted whereas other inhabitants of the island gradually returned to a religious practice of Islam that they had previously given up. This is especially true of the Palestinian shopkeepers, who had settled in the island in the early forties. The issue of roots and the memory of places play a prominent role in the attempt to organize Islam in Martinique as well as in the division of various Islamic groups. This paper analyzes three antagonistic references which variously affect believers' strategy and choices as far as places of worship and rituals are concerned: Arab identity and the reference to a Middle Eastern land of origin, the ambiguous reference to Africa, and finally, the reference to the Caribbean territory. 